\title{
A logarithmically completely monotonic function involving the gamma function and originating from the Catalan numbers
}

\author{
Fang-Fang Liu*, Xiao-Ting Shi, Feng Qi \\ Department of Mathematics, College of Science, Tianjin Polytechnic University, Tianjin City, 300387, China \\ *Corresponding author e-mail: fang-liu@qq.com
}

\begin{abstract}
Copyright (C)2015 Fang-Fang Liu, Xiao-Ting Shi, Feng Qi. This is an open access article distributed under the Creative Commons Attribution License, which permits unrestricted use, distribution, and reproduction in any medium, provided the original work is properly cited.
\end{abstract}

\begin{abstract}
In the paper, the author finds necessary and sufficient conditions for a function involving the gamma function and originating from investigation of properties of the Catalan numbers in combinatorics to be logarithmically completely monotonic.
\end{abstract}

Keywords: necessary and sufficient condition; logarithmically completely monotonic function; gamma function; Catalan number

\section{Introduction}

It is known [24] that, in combinatorics, the Catalan numbers $C_{n}$ for $n \geq 0$ form a sequence of natural numbers that occur in tree enumeration problems such as "In how many ways can a regular $n$-gon be divided into $n-2$ triangles if different orientations are counted separately?" The solution is the Catalan number $C_{n-2}$. The first few Catalan numbers $C_{n}$ for $0 \leq n \leq 11$ are

$1, \quad 1, \quad 2, \quad 5, \quad 14, \quad 42, \quad 132, \quad 429, \quad 1430, \quad 4862, \quad 16796, \quad 58786$.

Explicit formulas of $C_{n}$ for $n \geq 0$ include

$C_{n}=\frac{1}{n+1}\left(\begin{array}{c}2 n \\ n\end{array}\right)=\frac{(2 n) !}{n !(n+1) !}=\frac{2^{n}(2 n-1) ! !}{(n+1) !}=(-1)^{n} 2^{2 n+1}\left(\begin{array}{c}\frac{1}{2} \\ n+1\end{array}\right)=\frac{1}{n}\left(\begin{array}{c}2 n \\ n-1\end{array}\right)={ }_{2} F_{1}(1-n,-n ; 2 ; 1)$

and

$C_{n}=\frac{4^{n} \Gamma(n+1 / 2)}{\sqrt{\pi} \Gamma(n+2)}$,

where

$\Gamma(z)=\int_{0}^{\infty} t^{z-1} e^{-t} \mathrm{~d} t, \quad \Re(z)>0$ 
is the classical Euler gamma function and

${ }_{p} F_{q}\left(a_{1}, \ldots, a_{p} ; b_{1}, \ldots, b_{q} ; z\right)=\sum_{n=0}^{\infty} \frac{\left(a_{1}\right)_{n} \cdots\left(a_{p}\right)_{n}}{\left(b_{1}\right)_{n} \cdots\left(b_{q}\right)_{n}} \frac{z^{n}}{n !}$

is the generalized hypergeometric series defined for complex numbers $a_{i} \in \mathbb{C}$ and $b_{i} \in \mathbb{C} \backslash\{0,-1,-2, \ldots\}$, for positive integers $p, q \in \mathbb{N}$, and in terms of the rising factorials

$(x)_{n}= \begin{cases}x(x+1)(x+2) \cdots(x+n-1), & n \geq 1, \\ 1, & n=0 .\end{cases}$

The asymptotic form for the Catalan numbers is

$C_{x} \sim \frac{4^{x}}{\sqrt{\pi}}\left(x^{-3 / 2}-\frac{9}{8} x^{-5 / 2}+\frac{145}{128} x^{-7 / 2}+\cdots\right)$.

For more detailed information on the Catalan numbers $C_{n}$, please refer to the monographs $[1,2]$ and references therein.

In the paper [23], motivated by the explicit expression (1) and by virtue of an integral representation of the gamma function $\Gamma(x)$, the authors established an integral representation of the Catalan numbers $C_{x}$ for $x \geq 0$.

Theorem 1 ([23, Theorem 1]). For $x \geq 0$, we have

$C_{x}=\frac{e^{3 / 2} 4^{x}(x+1 / 2)^{x}}{\sqrt{\pi}(x+2)^{x+3 / 2}} \exp \left[\int_{0}^{\infty} \beta(t)\left(e^{-t / 2}-e^{-2 t}\right) e^{-x t} \mathrm{~d} t\right]$,

where

$\beta(t)=\frac{1}{t}\left(\frac{1}{e^{t}-1}-\frac{1}{t}+\frac{1}{2}\right)$

Recall from [4, Chapter XIII], [22, Chapter 1], and [25, Chapter IV] that an infinitely differentiable function $f$ is said to be completely monotonic on an interval $I$ if it satisfies

$0 \leq(-1)^{k} f^{(k)}(x)<\infty$

on $I$ for all $k \geq 0$. Recall from $[9,10]$ that an infinitely differentiable and positive function $f$ is said to be logarithmically completely monotonic on an interval $I$ if

$0 \leq(-1)^{k}[\ln f(x)]^{(k)}<\infty$

hold on $I$ for all $k \in \mathbb{N}$. For more information on logarithmically completely monotonic functions, please refer to [11, 12, 15, 21].

The formula (2) can be rearranged as

$\ln \left[\frac{\sqrt{\pi}(x+2)^{x+3 / 2}}{e^{3 / 2} 4^{x}(x+1 / 2)^{x}} C_{x}\right]=\int_{0}^{\infty} \beta(t)\left(e^{-t / 2}-e^{-2 t}\right) e^{-x t} \mathrm{~d} t$.

Since the function $\beta(t)$ is positive on $(0, \infty)$, see $[3,16,26]$ and references therein, the right hand side of $(3)$ is a completely monotonic function on $(0, \infty)$. This means that the function

$\frac{(x+2)^{x+3 / 2}}{4^{x}(x+1 / 2)^{x}} C_{x}$

is logarithmically completely monotonic on $(0, \infty)$. Because any logarithmically completely monotonic function must be completely monotonic, see [12, Eq. (1.4)] and references therein, the function (4) is also completely monotonic on $(0, \infty)$.

By virtue of (1), the function (4) can be rewritten as

$\frac{(x+2)^{x+3 / 2} \Gamma(x+1 / 2)}{(x+1 / 2)^{x} \Gamma(x+2)}, \quad x>0$.

Hence, the logarithmically complete monotonicity of (4) implies the logarithmically complete monotonicity of (5). The function (5) is a special case $F_{1 / 2,2}(x)$ of the general function

$F_{a, b}(x)=\frac{\Gamma(x+a)}{(x+a)^{x}} \frac{(x+b)^{x+b-a}}{\Gamma(x+b)}, \quad a, b \in \mathbb{R}, \quad a \neq b \quad x>-\min \{a, b\}$.

It is clear that

$F_{a, b}(x) F_{b, a}(x)=\left(\frac{x+b}{x+a}\right)^{b-a}=\left(1+\frac{b-a}{x+a}\right)^{b-a}$.

It is noted that the function $F_{a, b}(x)$ does not appear in the expository and survey articles $[6,7,12,13,14]$ and plenty of references therein. Therefore, it is significant to naturally pose an open problem below. 
Open Problem 1 ([23, Open Problem 1]). What are the necessary and sufficient conditions on $a, b \in \mathbb{R}$ such that the function $F_{a, b}(x)$ defined by $(6)$ is (logarithmically) completely monotonic in $x \in(-\min \{a, b\}, \infty)$ ?

The aim of this paper is to give solutions to the above open problem.

Theorem 2. The sufficient conditions on $a, b$ for the function $\left[F_{a, b}(x)\right]^{ \pm 1}$ defined by (6) to be logarithmically completely monotonic in $x \in(-\min \{a, b\}, \infty)$ are $(a, b) \in D_{ \pm}(a, b)$, where

$D_{+}(a, b)=\{a \geq 1, a>b\} \cup\left\{a \leq \frac{1}{2}, a<b\right\}$

and

$D_{-}(a, b)=\{a \geq 1, b>a\} \cup\left\{a \leq \frac{1}{2}, b<a\right\}$.

The necessary conditions on $a, b$ for the function $\left[F_{a, b}(x)\right]^{ \pm 1}$ to be logarithmically completely monotonic in $x \in(-\min \{a, b\}, \infty)$ are

$a(a-b) \gtreqless \frac{a-b}{2}$.

\section{Proof of Theorem 2}

Taking the logarithm of $F_{a, b}(x)$ gives

$$
\begin{aligned}
\ln F_{a, b}(x) & =\ln \Gamma(x+a)-x \ln (x+a)-[\ln \Gamma(x+b)-(x+b-a) \ln (x+b)] \\
& \triangleq f_{a}(x)-f_{a}(x+b-a) .
\end{aligned}
$$

Differentiating with respect to the variable $x$ of $f_{a}(x)$ yields

$f_{a}^{\prime}(x)=\psi(x+a)-\ln (x+a)+\frac{a}{x+a}-1$

and

$f_{a}^{\prime \prime}(x)=\psi^{\prime}(x+a)-\frac{1}{x+a}-\frac{a}{(x+a)^{2}}$.

In [8, Theorem 1.3], it was found that

1. the function $\psi(x)-\ln x+\frac{\alpha}{x}$ is completely monotonic on $(0, \infty)$ if and only if $\alpha \geq 1$,

2. the function $\ln x-\frac{\alpha}{x}-\psi(x)$ is completely monotonic on $(0, \infty)$ if and only if $\alpha \leq \frac{1}{2}$.

This means that

1. if $\alpha \geq 1$, the function $\frac{1}{x}+\frac{\alpha}{x^{2}}-\psi^{\prime}(x)$ is completely monotonic on $(0, \infty)$;

2. if $\alpha \leq \frac{1}{2}$, the function $\psi^{\prime}(x)-\frac{1}{x}-\frac{\alpha}{x^{2}}$ is completely monotonic on $(0, \infty)$.

Equivalently,

1. if $a \geq 1$, the function $-f_{a}^{\prime \prime}(x-a)$ is completely monotonic on $(0, \infty)$;

2. if $a \leq \frac{1}{2}$, the function $f_{a}^{\prime \prime}(x-a)$ is completely monotonic on $(0, \infty)$.

Consequently,

1. if $a \geq 1$, the function $-f_{a}^{\prime \prime}(x)$ is completely monotonic on $(-a, \infty)$;

2 . if $a \leq \frac{1}{2}$, the function $f_{a}^{\prime \prime}(x)$ is completely monotonic on $(-a, \infty)$.

As a result,

1. when $a \geq 1$ and $b-a>0$ the negativity of the function

$\left[\ln F_{a, b}(x)\right]^{\prime \prime}=f_{a}^{\prime \prime}(x)-f_{a}^{\prime \prime}(x+b-a)$

is completely monotonic on $(-a, \infty)$;

2. when $a \geq 1$ and $b-a<0$, the function $\left[\ln F_{a, b}(x)\right]^{\prime \prime}$ is completely monotonic on $(-b, \infty)$;

3. when $a \leq \frac{1}{2}$ and $b-a>0$, the function $\left[\ln F_{a, b}(x)\right]^{\prime \prime}$ is completely monotonic on $(-a, \infty)$;

4. when $a \leq \frac{1}{2}$ and $b-a<0$, the function $-\left[\ln F_{a, b}(x)\right]^{\prime \prime}$ is completely monotonic on $(-b, \infty)$.

In conclusion, 
1. when $(a, b) \in D_{+}(a, b)$, the function $\left[\ln F_{a, b}(x)\right]^{\prime \prime}$ is completely monotonic on $(-\min \{a, b\}, \infty)$;

2. when $(a, b) \in D_{-}(a, b)$, the function $-\left[\ln F_{a, b}(x)\right]^{\prime \prime}$ is completely monotonic on $(-\min \{a, b\}, \infty)$.

By straightforward computation, we see that

$$
\begin{aligned}
\lim _{x \rightarrow \infty}\left[\ln F_{a, b}(x)\right]^{\prime} & =\lim _{x \rightarrow \infty}\left[\psi(x+a)-\ln (x+a)+\frac{a}{x+a}-1-\psi(x+b)+\ln (x+b)-\frac{a}{x+b}+1\right] \\
& =\lim _{x \rightarrow \infty}\left[\psi(x+a)-\psi(x+b)+\ln \frac{x+b}{x+a}+\frac{a(b-a)}{(x+a)(x+b)}\right] \\
& =0 .
\end{aligned}
$$

This implies that, when $(a, b) \in D_{ \pm}(a, b)$, the first logarithmic derivative satisfies $\left[\ln F_{a, b}(x)\right]^{\prime} \lessgtr 0$. By the definition of logarithmically completely monotonic functions, we conclude that, when $(a, b) \in D_{ \pm}(a, b)$, the function $\left[F_{a, b}(x)\right]^{ \pm 1}$ is logarithmically completely monotonic on $(-\min \{a, b\}, \infty)$.

Conversely, if the function $\left[F_{a, b}(x)\right]^{ \pm 1}$ is logarithmically completely monotonic on $(-\min \{a, b\}, \infty)$, then $\pm\left[\ln F_{a, b}(x)\right]^{\prime} \leq 0$ which is equivalent to

$\psi(x+a)-\psi(x+b)+\ln \frac{x+b}{x+a}+\frac{a(b-a)}{(x+a)(x+b)} \lesseqgtr 0$.

This can be rearranged as

$a(a-b) \gtreqless(x+a)(x+b)\left[\psi(x+a)-\psi(x+b)+\ln \frac{x+b}{x+a}\right] \rightarrow \frac{a-b}{2}$

as $x \rightarrow \infty$. Therefore, the necessary conditions are $a(a-b) \gtreqless \frac{a-b}{2}$. The proof of Theorem 2 is complete.

Remark 1. About recent development on investigation of the function (7), please refer to the manuscript [17] and plenty of references therein.

Remark 2. This paper is a companion of the articles [18, 19, 20, 23] and a slightly revised version of the preprint [5].

\section{References}

[1] L. Comtet, Advanced Combinatorics: The Art of Finite and Infinite Expansions, Revised and Enlarged Edition, D. Reidel Publishing Co., Dordrecht and Boston, 1974.

[2] R. L. Graham, D. E. Knuth, and O. Patashnik, Concrete Mathematics - A Foundation for Computer Science, 2nd ed., Addison-Wesley Publishing Company, Reading, MA, 1994.

[3] A.-Q. Liu, G.-F. Li, B.-N. Guo, and F. Qi, Monotonicity and logarithmic concavity of two functions involving exponential function, Internat. J. Math. Ed. Sci. Tech. 39 (2008), no. 5, 686-691; Available online at http://dx.doi.org/10.1080/ 00207390801986841.

[4] D. S. Mitrinović, J. E. Pečarić, and A. M. Fink, Classical and New Inequalities in Analysis, Kluwer Academic Publishers, Dordrecht-Boston-London, 1993; Available online at http://dx.doi.org/10.1007/978-94-017-1043-5.

[5] F. Qi, A logarithmically completely monotonic function involving the gamma function and originating from the Catalan numbers, ResearchGate Research, available online at http://dx.doi.org/10.13140/RG.2.1.1401.2009.

[6] F. Qi, Bounds for the ratio of two gamma functions, J. Inequal. Appl. 2010 (2010), Article ID 493058, 84 pages; Available online at http://dx.doi.org/10.1155/2010/493058.

[7] F. Qi, Bounds for the ratio of two gamma functions: from Gautschi's and Kershaw's inequalities to complete monotonicity, Turkish J. Anal. Number Theory 2 (2014), no. 5, 152-164; Available online at http://dx.doi.org/10.12691/ tjant-2-5-1.

[8] F. Qi, Three classes of logarithmically completely monotonic functions involving gamma and psi functions, Integral Transforms Spec. Funct. 18 (2007), no. 7, 503-509; Available online at http://dx.doi.org/10.1080/10652460701358976.

[9] F. Qi and C.-P. Chen, A complete monotonicity property of the gamma function, J. Math. Anal. Appl. 296 (2004), 603-607; Available online at http://dx.doi.org/10.1016/j.jmaa.2004.04.026.

[10] F. Qi and B.-N. Guo, Complete monotonicities of functions involving the gamma and digamma functions, RGMIA Res. Rep. Coll. 7 (2004), no. 1, Art. 8, 63-72; Available online at http://rgmia.org/v7n1.php.

[11] F. Qi, S. Guo, and B.-N. Guo, Complete monotonicity of some functions involving polygamma functions, J. Comput. Appl. Math. 233 (2010), no. 9, 2149-2160; Available online at http://dx.doi.org/10.1016/j.cam.2009.09.044.

[12] F. Qi and W.-H. Li, A logarithmically completely monotonic function involving the ratio of gamma functions, J. Appl. Anal. Comput. 5 (2015), no. 4, 626-634; Available online at http://dx.doi.org/10.11948/2015049. 
[13] F. Qi and Q.-M. Luo, Bounds for the ratio of two gamma functions-From Wendel's and related inequalities to logarithmically completely monotonic functions, Banach J. Math. Anal. 6 (2012), no. 2, 132-158.

[14] F. Qi and Q.-M. Luo, Bounds for the ratio of two gamma functions: from Wendel's asymptotic relation to ElezovićGiordano-Pečarić's theorem, J. Inequal. Appl. 2013, 2013:542, 20 pages; Available online at http://dx.doi.org/10. $1186 / 1029-242 \mathrm{X}-2013-542$.

[15] F. Qi, Q.-M. Luo, and B.-N. Guo, Complete monotonicity of a function involving the divided difference of digamma functions, Sci. China Math. 56 (2013), no. 11, 2315-2325; Available online at http://dx.doi.org/10.1007/ s11425-012-4562-0.

[16] F. Qi, Q.-M. Luo, and B.-N. Guo, The function $\left(b^{x}-a^{x}\right) / x$ : Ratio's properties, In: Analytic Number Theory, Approximation Theory, and Special Functions, G. V. Milovanović and M. Th. Rassias (Eds), Springer, 2014, pp. 485-494; Available online at http://dx.doi.org/10.1007/978-1-4939-0258-3_16.

[17] F. Qi, F.-F. Liu, and X.-T. Shi, Comments on two completely monotonic functions involving the q-trigamma function, ResearchGate Dataset, available online at http://dx.doi.org/10.13140/RG.2.1.3267.4401.

[18] F. Qi, X.-T. Shi, and F.-F. Liu, An exponential representation for a function involving the gamma function and originating from the Catalan numbers, ResearchGate Research, available online at http://dx.doi.org/10.13140/RG.2.1. 1086.4486.

[19] F. Qi, X.-T. Shi, and F.-F. Liu, An integral representation, complete monotonicity, and inequalities of the Catalan numbers, ResearchGate Technical Report, available online at http://dx.doi.org/10.13140/RG.2.1.3230.1927.

[20] F. Qi, X.-T. Shi, and F.-F. Liu, Several formulas for special values of the Bell polynomials of the second kind and applications, ResearchGate Technical Report, available online at http://dx.doi.org/10.13140/RG.2.1.3230.1927.

[21] F. Qi, C.-F. Wei, and B.-N. Guo, Complete monotonicity of a function involving the ratio of gamma functions and applications, Banach J. Math. Anal. 6 (2012), no. 1, 35-44; Available online at http://dx.doi.org/10.15352/bjma/ 1337014663.

[22] R. L. Schilling, R. Song, and Z. Vondraček, Bernstein Functions-Theory and Applications, 2nd ed., de Gruyter Studies in Mathematics 37, Walter de Gruyter, Berlin, Germany, 2012; Available online at http://dx.doi.org/10.1515/ 9783110269338.

[23] X.-T. Shi, F.-F. Liu, and F. Qi, An integral representation of the Catalan numbers, ResearchGate Research, available online at http://dx.doi.org/10.13140/RG.2.1.2273.6485.

[24] R. Stanley and E. W. Weisstein, Catalan Number, From MathWorld-A Wolfram Web Resource; Available online at http://mathworld.wolfram.com/CatalanNumber.html.

[25] D. V. Widder, The Laplace Transform, Princeton Mathematical Series 6, Princeton University Press, Princeton, N. J., 1941.

[26] S.-Q. Zhang, B.-N. Guo, and F. Qi, A concise proof for properties of three functions involving the exponential function, Appl. Math. E-Notes 9 (2009), 177-183. 